\title{
Schwannoma in Masquerade
}

\author{
Shaji Thomas $\cdot$ N. Vezhavendan $\cdot$ V. Yuvaraj · \\ Gagan Thakur $\cdot$ V. Dal Singh
}

Received: 7 August 2009/Accepted: 24 January 2011/Published online: 18 March 2011

(C) Association of Oral and Maxillofacial Surgeons of India 2011

\begin{abstract}
Neurilemmoma is a rare nerve tumor seldom included in the list of differential diagnosis of soft tissue swelling in maxillofacial region. Arrival at a clinical diagnosis of schwannomas is rarely possible as there are hardly any characteristic clinical features to help in its and it is mostly established by histopathologic examination. We report an interesting case which had presentations similar to a sebaceous cyst but finally turned out to be a schwannomma.
\end{abstract}

Keywords Neurilemmoma $\cdot$ Schwannoma $\cdot$ Lemmoma

\section{Introduction}

Neurilemmomas are benign, encapsulated tumors of the nerve sheath. Their cells of origin are thought to be Schwann cells derived from the neural crest. Verocay was the first to describe these lesions in 1908 and named them neurinomas. Since its the initial report, the tumor has been given a number of names, including solitary nerve sheath tumor, perineural fibroblast tumor, neurilemmomas and schwannoma [1]. Approximately $25-40 \%$ of all neurilemmomas are seen in the head and neck region, and only

S. Thomas · N. Vezhavendan · V. Yuvaraj · G. Thakur ·

V. Dal Singh

Department of OMFS, Peoples College of Dental Sciences,

Bhopal, India

e-mail: getyuva@hotmail.com

G. Thakur

e-mail: drgagan05@yahoo.com

S. Thomas $(\bowtie)$

A/160, new minal residency, J. k road, Govindpura, Bhopal 462

023, India

e-mail: shajihoss@gmail.com
$1 \%$ exhibits an intraoral location [2, 3]. Though it can occur at any age it is more common in persons aged 20-50. Most of the reports indicate a 2:1 female predilection [4]. The differential diagnosis of a benign tumor of soft tissue includes sebaceous cyst, neurofibroma, ganglion cyst, giant cell tumor of tendon sheath etc. In this article we report a case of a swelling in the upper lip which had similar presentations of a sebaceous cyst, which turned out to be a schwannoma on histopathologic examination.

\section{Case Report}

A 24 years old woman reported to our clinic with a complaint of a swelling measuring around $1 \times 1 \mathrm{~cm}$ on the left side of face near left naso-labial fold, with one year duration. It was not associated with pain or paresthesia. Skin over the swelling was discolored and a darkened spot which appear as a punctum was noticed (Fig. 1). The swelling was not warm and was firm in consistency. Skin over the swelling was not freely mobile. She also gave history of pus discharge in the past from the same region. A physical examination conducted on the patient failed to reveal similar lesions anywhere on her body. Her medical history was unremarkable. Radiographic examination was not significant. A provisional diagnosis of sebaceous cyst was arrived at. To avoid an extra oral scar, excision of the lesion through an intraoral approach was planned. Excisional biopsy was performed under local anesthesia. Absence of attachment of the lesion to punctum, clean cleavage plane around the lesion and presence of a thick capsule were the significant surgical findings (Fig. 2). The lesion was dissected out from the subcutaneous plane in toto and submitted for histopathologic examination. 


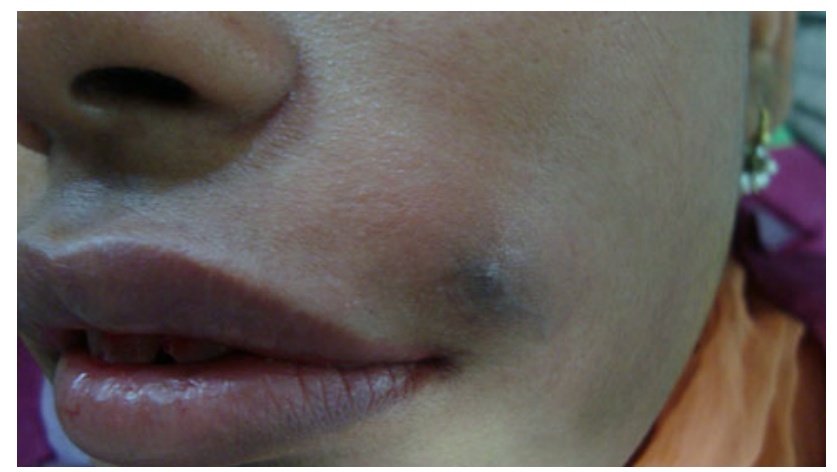

Fig. 1 Clinical picture showing discoloured skin with punctum

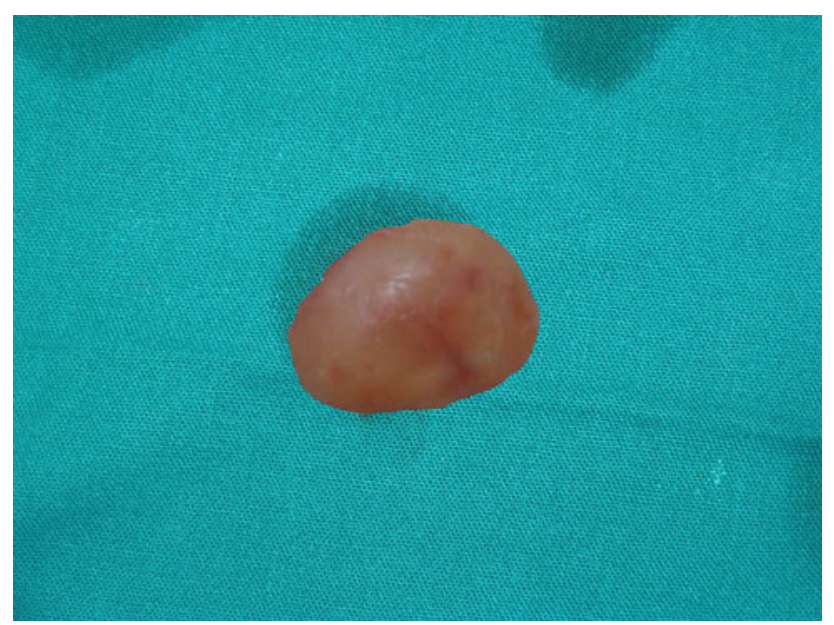

Fig. 2 Excised lesion

\section{Discussion}

Based on the clinical findings like location of the swelling, presence of punctum and occasional discharge, we assumed the clinical diagnosis to be a sebaceous cyst; but to our surprise the histopathologic picture showed a well capsulated lesion with mixture of Antoni A along with Verocay bodies and Antoni B patterns which were pathonomic features of schwannomas [5, 6] (Figs. 3, 4). By virtue of wisdom we were unable to rule out the possibility of acne, which could mimic as sebaceous cyst. Most of the literature suggests that clinical course and the radiographic appearance of neurilemmoma is not of significance, therefore clinical diagnosis is not possible and can only be established on histologic examination [7, 8]. Clinically and histologically, schwannoma should be differentiated from neurofibroma, which is a more common neurogenic tumor consisting of a mixture of Schwann cells and fibroblasts. Neurofibroma is rarely encapsulated and often present in a multiple fashion and in conjunction with neurofibromatosis (type 2). Because neurofibroma shows a much greater propensity for malignant transformation, it is important to

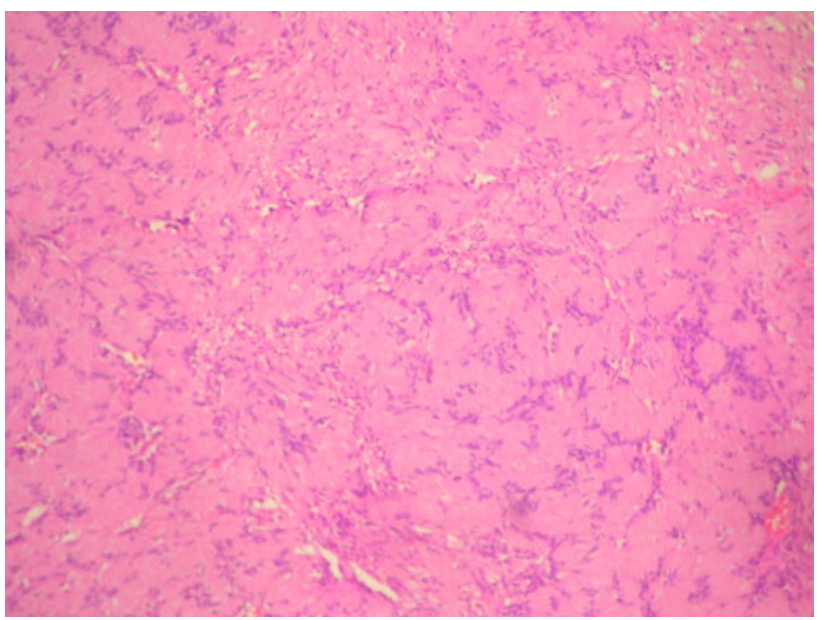

Fig. 3 Photomicrogrphic picture. H\&E staining, $10 \times$ magnification

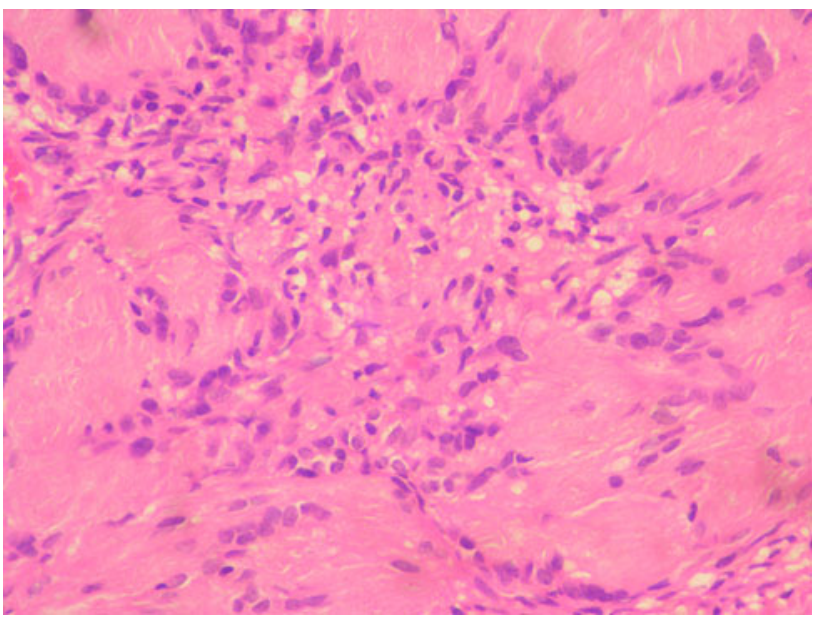

Fig. 4 Photomicrogrphic picture. H\&E staining, $40 \times$ magnification

differentiate it from schwannomas [9]. In younger patients, as in our case, neurilemmoma should be distinguished from neurofibromatosis type 2 (NF2)- associated schwannomas $(7,8)$ that are composed of neoplastic Schwann cells, but which differ from sporadic schwannomas in a number of ways. NF2 patients usually develop bilateral vestibular schwannomas and have higher proliferative activity. NF2 schwannomas may appear multilobular on both gross and microscopic examination.

In a series of 303 cases reported by Das Gupta [10] and his associates only 16 cases where found to be in buccal mucosa and lip. In a study of 41 tumors Oberman [11] and Sulliger reported that $25 \%$ were located inside the mouth. Hatziotis et al. [12] in a review of intraoral neurilemmomas, found only 7 in lip and 6 in buccal mucosa. Although neurilemmomas are usually asymptomatic neoplasm, cases accompanied by pain and paresthesia have been reported. Surgical excision is the treatment of choice. Complete 
excision, however, has a good prognosis due to the low recurrence rate and the rarity of malignant transformation. Our experience also stresses on the fact that all tissue excised should be subjected to histopathologic examination to avoid missing such rare lesions in masquerade.

\section{Conflict of interest None.}

\section{References}

1. Clifton MA (1977) Sympathetic neurilemmoma: an uncommon cause of solitary cervical swelling. J R Coll Surg Edinb 22: 351-354

2. Harada H, Omura K, Maeda A (2001) Amassive pleomorphic adenoma of the submandibular salivart gland accompanied by neurilemmoma of the neck misdiagnosed as a malignant tumor: report of a case. J Oral Maxillofac Surg 59:931-935

3. Pfeifle R, Baur DA, Paulino A et al (2001) Schwannoma of the tongue:report of 2 cases. J Oral Maxillofac Surg 59:802-804
4. Pimkhaokham A et al (2006) Central neurilemmoma of the jaw in concurrence with radicular cyst: a case report. Oral Surg Oral Med Oral Path Oral Radiol Endod 102:34-36

5. Zachariades N (1984) Schwannoma of the oral cavity. Review of the literature and report of a case. J Oral Med 39:41-43

6. Shimura K, Allen EC, Kinoshita Y et al (1973) Central neurilemmoma of the mandible: Review of the literature and report of a case. J Oral Surg 31:363

7. Eversole LR (1969) Central benign and malignant neural neoplasm of the jaws: a Review. J Oral Surg 27:716

8. Rengaswamy V (1978) Central neurilemmoma of the jaws. Review of the literature and a case report. Int J Oral Maxillfac Surg 7:300

9. Geist JR, Gander DL, Stefanac Sj (1992) Oral manifestation of neurofibromatosis types I and II. Oral Surg Oral Med Oral Path Oral Radiol Endod 73:376-382

10. Das Gupta TK et al (1969) Beningn solitary schwannoma. Cancer $24: 355$

11. Oberman H, Sullenger G (1967) Neurogenous tumor of the head and neck. Cancer 20:1992-2001

12. Hatziotis J, Asprides H (1989) Neurilemmoma of the mandible:report of a case. Br J Oral Maxillofac Surg 27:512-516 